\title{
TOWARDS AN ALGORITHM FOR NEAR REAL TIME PROFILING OF AEROSOL SPECIES, TRACE GASES, AND CLOUDS BASED ON THE SYNERGY OF REMOTE SENSING INSTRUMENTS
}

\author{
Nikolaos Siomos $^{1 *}$, Dimitrios Balis ${ }^{1}$, Alkiviadis Bais ${ }^{1}$, Mariliza Koukouli ${ }^{1}$, Katerina Garane ${ }^{1}$, Kalliopi \\ A. Voudouri ${ }^{1}$, Fani Gkertsi ${ }^{1}$, Athanasios Natsis ${ }^{1}$, Dimitrios Karagkiozidis ${ }^{1}$, Ilias Fountoulakis ${ }^{1,2}$ \\ ${ }^{1}$ Laboratory of Atmospheric optics, Laboratory of Atmospheric Physics, Physics Department, Aristotle \\ University of Thessaloniki, Greece \\ ${ }^{2}$ Aosta Valley Regional Environmental Protection Agency (ARPA), Saint-Christophe, Italy \\ *Email: nsiomos@physics.auth.gr
}

\begin{abstract}
In this manuscript we present the concept of a novel algorithmic chain that aims to a dataset of unprecedented detail in the vertical distribution of multiple atmospheric components in near real time conditions. The analysis will be based on the following remote sensing instruments: a depolarization Raman lidar, a visible and a thermal all-sky camera, a Brewer spectrophotometer, and up to three mini DOAS/MAX-DOAS systems. Based on both individual and synergistic processing of the data collected, novel products will be made available in near real time conditions to the end users. Columnar aerosol information from the spectrophotometers will be combined with lidar data to retrieve vertical profiles of individual aerosol species. Cloud layers will be detected and classified based mainly on the synergy of the lidar and the sky cameras and a realistic 3D representation of cloud conditions around the measurement site will be produced. Lidar profiles will be implemented as a priori information for radiative transfer purposes, that are necessary in order to obtain high quality trace gases profiles from the DOAS/MAX-DOAS spectrophotometer. Fast synergistic data processing will ensure that the algorithm can be applied for near real time public data dissemination in the future.
\end{abstract}

\section{INTRODUCTION}

Air quality forms an indisputable major global concern as in year 2014 only $12 \%$ of the Earth's urbanized population lived in cities whose air quality levels comply with the World Health Organization, WHO, guideline levels. Recent studies show that more people die because of air pollution than malaria and HIV combined, including 1.4 million people in China, 650000 in India and 180000 in Europe, per annum. Accurate information on air quality levels traditionally depends on local monitoring stations. Their task is to monitor multiple atmospheric components. These components can be summarized in four main categories: aerosol species, clouds, trace gases, and greenhouse gases. Since they are of quite different nature, variable vertical distribution, and also considering inherent difficulties in performing in situ measurements in high altitudes, it is rather challenging for a single station to provide a complete, spatially resolved dataset. While the use of remote sensing techniques overcomes difficulties such as targeting the elevated atmospheric components, the retrievals from single instruments typically leads to limited datasets. If a holistic approach is desired, synergies between remote sensing instrumental techniques are certainly required. The need for such datasets is also evident in radiative transfer modeling, considering that all atmospheric components interact with radiation.

This study proposes new potential synergies using a network of co-located remote sensing instruments installed in the facilities of the Laboratory of Atmospheric Physics, in Thessaloniki, Greece $(40.63,22.95,60 \mathrm{~m})$ : a depolarization Raman lidar, a visible and a thermal all-sky cameras, a Brewer spectrophotometer, and up to three mini DOAS/MAX-DOAS systems. The main goal is to provide the algorithmic means to reveal new information on atmospheric components. A prototype chain of processing algorithms that will operate with standard streams and perform automated inversions, will allow fast monitoring of the atmospheric components and their effect on radiation that is really hard to achieve otherwise. 


\section{INSTRUMENTATION}

Multiple instruments that monitor the atmosphere are currently installed in the facilities of the Laboratory of Atmospheric Physics. They are constantly maintained and calibrated, and most of them have participated in international field campaigns. The current equipment includes:

- A double-monochromator Brewer spectrophotometer that performs parallel direct and global spectral measurements of the solar irradiance in the $290-363 \mathrm{~nm}$ wavelength range since 1997. Information on the calibration and QA/QC of the measured spectra can be found in [1] and [2]. It will provide aerosol properties such as the spectral UV Aerosol Optical Depth (AOD) and Single Scattering Albedo (SSA).

- A multiwavelength depolarization Raman lidar that is installed in LAP-AUTH since 2000. The current setup of the system includes three elastic channels at 355, 532 and $1064 \mathrm{~nm}$, two nighttime only Raman channels at 387 and $607 \mathrm{~nm}$ and two polarization channels (parallel and cross) at 532 $\mathrm{nm}$. A detailed description of the system can be found in [3]. It will provide aerosol properties such as the backscatter, depolarization, and extinction profiles, and also cloud properties such as the cloud boundaries, optical depth, and the ice/ water phase at zenith.

- Three mini DOAS/MAX-DOAS spectrophotometers that perform automated daytime measurements in the wavelength range $297-450 \mathrm{~nm}$ of direct solar irradiance and diffuse sky radiance since 2013. They consist of a cooled, miniature CCD spectrograph and a 2-axes tracker. They are described in [4]. They will provide trace gases' concentrations $\left(\mathrm{NO}_{2}, \mathrm{HCHO}, \mathrm{SO}_{2}, \mathrm{O}_{4}\right.$, and $\mathrm{O}_{3}$ ) and also aerosol properties such as the daytime spectral UV AOD, UV SSA and the Asymmetry Factor as well as the nighttime spectral UV AOD.

- A commercial digital all-sky camera with a fisheye lens and a hemispheric dome that is installed since 2012. The addition of a thermal all-sky camera in the longwave infrared region to the current equipment would allow nighttime cloud detection (e.g. [5]), extending synergies with the lidar after sunset. They will provide cloud properties such as the cloud fraction, mask, classification, brightness temperature, and the cloud optical depth (COD).

\section{METHODOLOGY}

A synopsis of the implementation of the research idea is presented here. Initially, measurements from all relevant instruments will be gathered in a pilot dataset. They will be analyzed towards their potential to reveal information on the vertical and spatial distribution of aerosol species, trace gases, and clouds. The necessary algorithms that link the raw data with the final retrievals will be implemented first. This includes relevant routine inversion algorithms already adopted by the station, state of the art algorithms that are going to be adjusted to the current equipment and novel synergistic techniques that will be developed in the frame of this project. All the individual modules will be integrated into the umbrella algorithm: Synergistic Profiling Algorithm for the Retrieval of Trace Gases, Aerosol Species, and ClOUdS (SPARTACUS), which will automatically process the raw data from the instruments and apply the necessary inversions, proceed to the synergistic algorithms, and finally combine the retrievals to a common output. It will follow a standard input/output protocol in order to facilitate implementation in other stations in the future. Finally, an Operational Acceptance Testing will ensure that all processes are robust enough for near real time application. The final products of SPARTACUS algorithm will include:

- Aerosol Species Concentration Profiles: A new algorithm will be designed for the retrieval of aerosol species concentration profiles based on the synergy of lidar profiles and spectrophotometer columnar aerosol properties. Aerosols will be modeled as mixtures of up to 9 aerosol species with distinct size distributions and dry refractive indices, and variable total concentration similar to the OPAC database [6]. The additional parametrizations of [7] will also be considered. The hygroscopic growth will be calculated using humidity profiles from meteorological models and/or radiosondes and the absorbed water concentration per species will also be retrieved. 


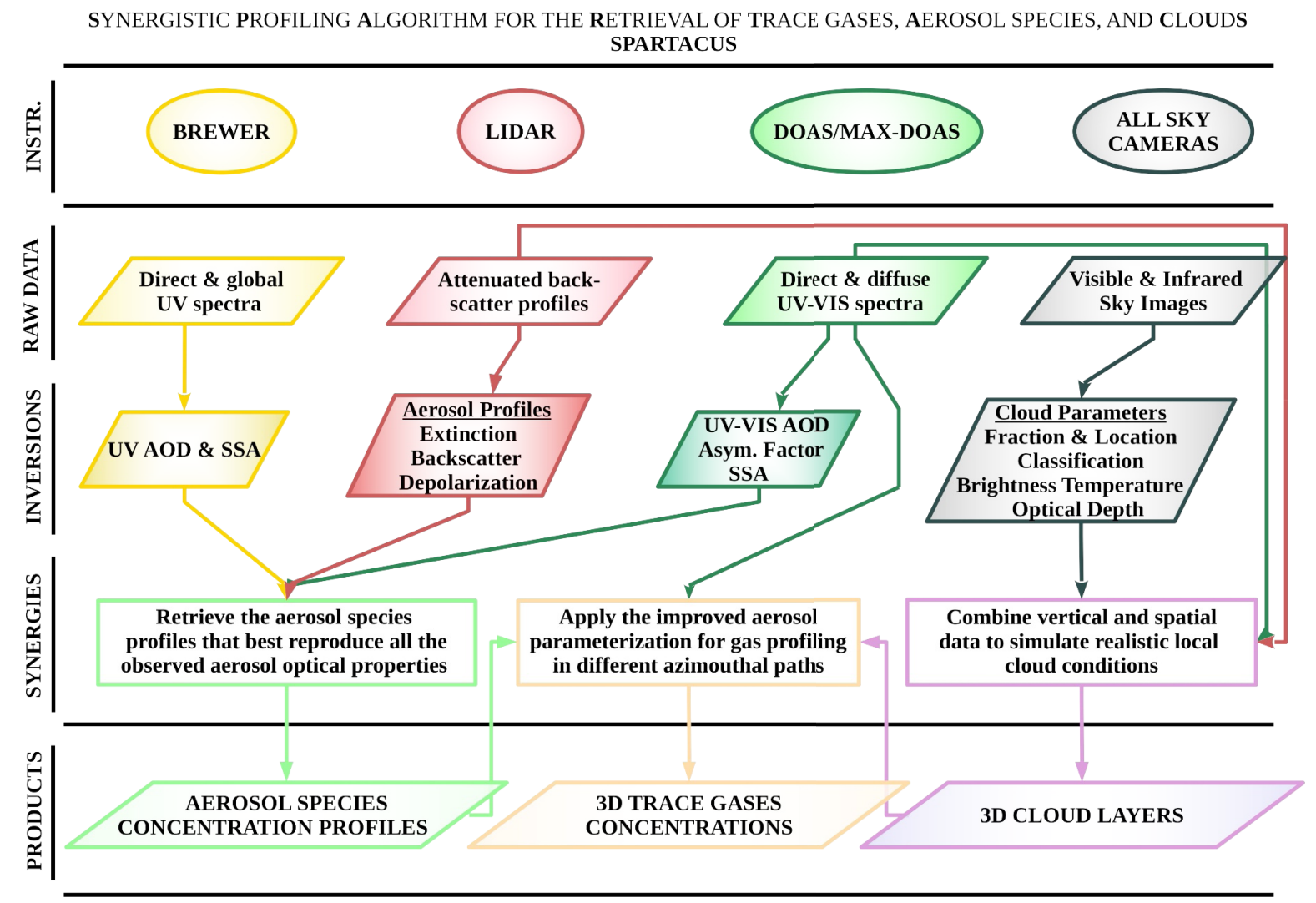

Figure 1: Flowchart of the umbrella algorithm SPARTACUS. Instruments are marked with round borders, algorithms with square boxes and products with parallelograms.

The species will include fine dust, coarse dust, fine sea salt, coarse sea salt, coarse volcanic ash, fine black carbon, fine carbonaceous, fine water soluble and potentially coarse pollen. During daytime, spectrophotometer columnar aerosol products will supplement lidar backscatter and depolarization profiles. During nighttime, the addition of the extinction profiles could suffice for a decent separation of some aerosol species (e.g. [7]). The potential to separate more species, though, by including lunar DOAS/MAX-DOAS spectral AOD will also be examined. Concerning the inversion method, look up table and optimal estimation approaches will be investigated. The first inversion category will focus on building a library of simulated aerosol mixtures and then apply machine learning techniques in order to identify the mixture that best describes the measurements. The latter will focus on building a regression algorithm (e.g. non linear least squares, total least squares) that will solve towards the concentration profiles combination that best reproduces the observations.
- 3D Trace Gases Concentration: Many techniques currently provide trace gas profiles from DOAS/MAX-DOAS systems (e.g. [8]) by applying an aerosol extinction profile, usually either from a climatology or from DOAS/MAXDOAS $\mathrm{O}_{4}$ measurements. In both cases, such a profile cannot compete with the high resolution and accuracy a dedicated lidar can offer. The application of the novel aerosol species concentration profiles will further improve the radiative transfer calculations that are required for the gas profiling due to the advanced aerosol parameterization which goes far beyond a simple extinction profile application. Both optimal estimation and lookup table approaches will be considered for the inversions. By measuring in different azimuthal paths, the possibility to derive the spatial distribution of traces gases around the instrument will also be examined. Finally, the novel cloud products can be applied in order to investigate the effect of partial cloud cover to the retrievals. 
- 3D Cloud Layers: A new algorithm designed for realistic simulation of cloud conditions will be developed based on lidar, all sky cameras, and DOAS/MAX-DOAS measurements. While a lidar can accurately and diurnally monitor cloud properties at zenith, it lacks information about their spatial distribution that can be diurnally provided by sky cameras. The placement of the two cameras could allow the application of stereophotometric techniques and the inference of cloud base height information (e.g [9]). Cloud base trigonometry with the DOAS/MAX-DOAS systems can also be investigated during daytime as there are currently three of them available. As cloud optical depth, base brightness temperature, and classified type can also be indicative of the cloud base height and thickness, they can be applied in order to improve the stereophotometric retrievals, also in combination with the accurate lidar zenith cloud products. Finally, a pattern recognition algorithm will identify continuous cloud layers from sky images and cluster them in categories based on similarity. This will determine whether it is safe to expand the detailed lidar information in zenith to other parts of the sky with similar cloud conditions.

\section{THE IMPACT}

The ability to obtain the vertical distribution of individual aerosol species and trace gases is still quite fresh in the scientific community. The atmospheric modeling community for sure would benefit the most as such information can be invaluable for the evaluation of forecasting models. The same products could be utilized in the evaluation of satellite observations as well. As cloud formation depends largely on the existing aerosol load and some aerosol species originate from trace gases, applications in the fields of aerosol and cloud interactions and aerosol formation in elevated layers are also possible. Since both aerosols and trace gases are factors that negatively affect public health, the knowledge of their concentration levels inside the boundary layer is important in preventing detrimental exposure by timely warning the public. Likewise, a near real time detection of extreme aerosol outbreaks, such as dust and volcanic ash plumes, can be critical in avoiding aviation hazards. Applications could even focus towards agriculture as there is evidence that aerosol species and trace gases could damage crops directly by being absorbed by the plants.

\section{ACKNOWLEDGMENTS}

We acknowledge support of this work by the project "PANhellenic infrastructure for Atmospheric Composition and climatE change" (MIS 5021516) which is implemented under the Action "Reinforcement of the Research and Innovation Infrastructure", funded by the Operational Programme "Competitiveness, Entrepreneurship and Innovation" (NSRF 20142020). This research is co-financed through the Operational Program «Human Resources Development, Education and Lifelong Learning 2014-2020» in the context of the project "Optimization and application of methods for ground-based remote sensing of aerosols and ozone in the lower troposphere for investigating their variability" (MIS 5004486). Kalliopi A. Voudouri acknowledges the support of the General Secretariat for Research and Technology and the Hellenic Foundation for Research and Innovation. Dimitrios Karagkiozidis and Fani Gkertsi acknowledge the support of the Operational Programme «Human Resources Development, Education and Lifelong Learning» in the project "Strengthening Human Resources Research Potential via Doctorate Research" (MIS5000432), implemented by the State Scholarships Foundation (IKY).

\section{REFERENCES}

[1] Kazadzis, et al. Applied Optics, 44(9), 16811690 (2005)

[2] Garane, et al. Ann. Geophys., 24(12), 32153228 (2006)

[3] Siomos, et al. Atmos. Chem. Phys., 18, 1188511903 (2018)

[4] Gkertsi, et al. Atmospheric Environment, 180, 51-58 (2018)

[5] Aebi, et al. Atmos. Meas. Tech., 11, 55495563, (2018)

[6] Hess, et al. Bulletin of the American Meteorological Society, 79, 831-844 (1998)

[7] Hara, et al. Remote Sensing, 10 (2018)

[8] Frieß, et al. Atmos. Meas. Tech. Discuss., in review (2018)

[9] Crispel, P. and Roberts, G. Atmos. Meas. Tech., 11, 593-609 (2018) 